\title{
Effect of whole body vibration on lactate level recovery and heart rate recovery in rest after intense exercise
}

\author{
Seung Rok Kang ${ }^{\mathrm{a}}$, Jin-Young Min ${ }^{\mathrm{b}}$, Changho $\mathrm{Yu}^{\mathrm{c}, *}$ and Tae-Kyu Kwon ${ }^{\mathrm{d}, *}$ \\ ${ }^{a}$ Department of Healthcare Engineering, Chonbuk National University, Jeonju, Jeonbuk 54896, Korea \\ ${ }^{\mathrm{b}}$ Corporation of Sonicworld, Wonju, Kwangwondo 26365, Korea \\ ${ }^{\mathrm{c}}$ Department of Convergence Technology Engineering, Chonbuk National University, Jeonju, Jeonbuk \\ 54896, Korea \\ ${ }^{\mathrm{d}}$ Division of Biomedical Engineering, Chonbuk National University, Jeonju, Jeonbuk 54896, Korea
}

\begin{abstract}
.
OBJECTIVE: In this paper, we investigated the recovery of the lactate level, muscular fatigue, and heart rate recovery (HRR) with respect to whole body vibration (WBV) during the rest stage after a gait exercise.

METHODS: A total of 24 healthy subjects with no medical history of exercise injury participated. The participants were divided into a training group with vibration during rest and a control group with the same conditions but without vibration. The subjects performed a gait exercise with a slope of $15 \%$ and velocity of $4 \mathrm{~km} / \mathrm{h}$ to consume $450 \mathrm{kcal}$ in $30 \mathrm{~min}$. Then, they rested on a vibrating chair or on a chair without vibrations for $30 \mathrm{~min}$. The vibration protocol consists of a frequency of $10 \mathrm{~Hz}$ and amplitude of $5 \mathrm{~mm}$. To estimate the recovery effect, we measured the lactate levels in blood, spectral edge frequency (SEF) of MVIC, and HRR before, immediately after exercise, and after rest.

RESULTS: The results showed that the lactate level in the training group decreased more (93.8\%) than in the control group $(32.8 \%)$. Also, HRR showed a similar trend with a recovery of $88.39 \%$ in the training group but $64.72 \%$ in the control group. We considered that whole-body vibrations during rest would help remove lactic acid by improving the level of lactic acid oxidation with stimulated blood vessels in the muscles and by helping to maintain blood flow. Also, WBV would lead to compensation to actively decrease the fast excess post-exercise oxygen consumption from blood circulation.

CONCLUSIONS: We suggest that whole-body vibrations during rest can provide fast, efficient fatigue recovery as a cool down exercise for women, the elderly, and patients without other activity after intense exercise.
\end{abstract}

Keywords: Fatigue, lactate level in blood, heart rate recovery, whole body vibration, healthcare

\section{Introduction}

Recently, there has been research and development of various exercise methods and machines as a result of interest in exercise for people of all ages to improve their health [1]. Whole body vibration has entered the limelight as a new exercise method in sports training and rehabilitation during the last

\footnotetext{
${ }^{*}$ Corresponding authors: Tae-Kyu Kwon, Division of Biomedical Engineering, Chonbuk National University, 567 Baekjedaero, Deokjin-gu, Jeonju-si, Jeonbuk 54896, Korea. Tel.: +82 632704066; Fax: +82 632702247; E-mail: kwon10@ jbnu.ac.kr and Changho Yu, Department of Convergence Technology Engineering, Chonbuk National University, 567 Baekje-daero, Deokjin-gu, Jeonju-si, Jeonbuk 54896, Korea. Tel.: +82 634722898; Fax: +82 632704226; E-mail: combo418@ nate.com.
}

0928-7329/17/\$35.00 (C) 2017 - IOS Press and the authors. All rights reserved

This article is published online with Open Access and distributed under the terms of the Creative Commons Attribution NonCommercial License (CC-BY-NC 4.0). 
decade [2,3]. Whole body vibration (WBV) provides harmless vibrations to the whole body including stimulated muscles $[4,5]$. Research on WBV has focused in the areas of sports and medical science. The effects of WBV include increased muscular strength, power, and jumping performance with muscular ability in untrained and elderly individuals, and in young trained subjects [6]. Other studies tested the effects of WBV on flexibility and they found positive effects [7,8]. Also, Park reported that WBV could change metabolic and physiological variables according to vibration frequency [9].

Positive effects of WBV have also been reported in rehabilitation, as well exercise effects. Also, there has been research in rehabilitation and treating elderly persons' diseases and preventing falls using WBV; positive effects on muscle strength, living ability, gait, and postural balance were reported [10,11]. Moreover, it was reported that WBV was helpful in osteoporosis, improving muscle strength and proprioception [12].

According to these effects, foundational studies on WBV have examined parameters such as frequency, amplitude, and duration [13]. WBV effects were accelerated by frequency and amplitude and stimulated human body exercise load [14,15]. A few studies reported significant effects of frequency or amplitude on muscle function $[16,17]$. Moreover, it was recently reported that WBV could provide a more efficient warm-up method to improve range of motion, compared with 'traditional' exercise methods, such as stretching [18-20].

To our knowledge, the WBV studies reported to date concern the effects on muscle function, flexibility, postural balance, and warm-up. There has been no study estimating the recovery effect, such as cooldown. Thus, this study was to investigate the recovery effect in terms of lactate level, medial frequency of muscular activity, and HRR according to WBV during rest after a gait exercise. We suggest that WBV could be an appropriate part of exercise cool-down.

\section{Methods}

\subsection{Subjects}

For this study, we recruited 24 healthy female subjects with no medical history related to the musculoskeletal system from exercise injury. They were divided into two groups, consisting of a training group and a control group. We provided the vibration during rest after a gait exercise in the training group (TG) and without vibration during rest in the control group (CG). Written informed consent conforming to the Declaration of Helsinki (1964) was obtained from all volunteers. After checking that no subject had any problem with WBV, we also checked for similar body weights, heights, life habits, and exercise habits.

\subsection{Chair-type vibrator}

All vibration and control protocols were performed on a developed vibrator. This platform produces up-to-down alternating vertical sinusoidal vibration in the chair using sonic waves. The vibrator could provide vibration with frequency from 10 to $50 \mathrm{~Hz}$ as 10 units, amplitude from 1 to $5 \mathrm{~mm}$ as 1 unit and duration time from 1 to $30 \mathrm{~min}$ as 1 unit. The locations of the vibration sources were eight spots: the shoulder blades, lumbar area, femoral area, and lower legs, left and right in Fig. 1(a).

\subsection{Experimental procedure}

We determined all subjects' ages, heights, and weights. They were divided into a training group with 


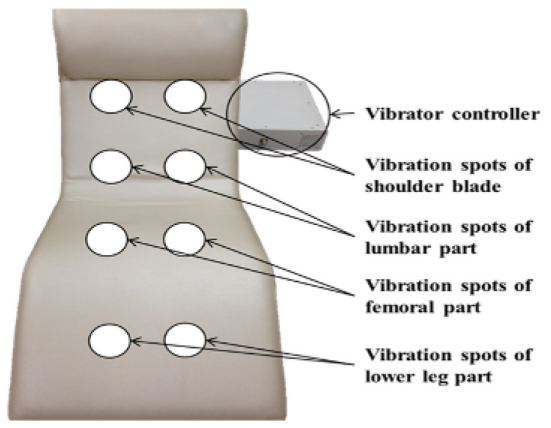

(a)

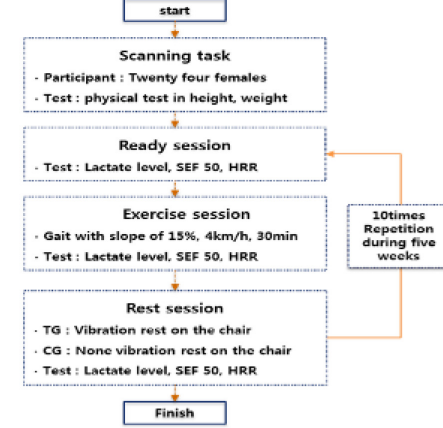

(b)

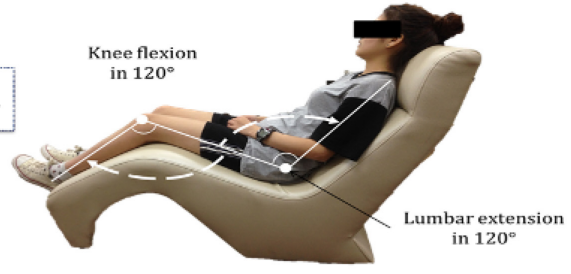

(c)

Fig. 1. (a) Vibrations provided were up-to-down alternating vertical sinusoidal vibration in the chair using sonic waves and vibration spots, (b) Experimental procedure per session according to whole body vibration (WBV), (c) Vibration posture with angles of knee flexion and lumbar extension during the rest session in the chair-type vibrator.

WBV and a control group without vibration. The experimental procedure progressed in sequence: ready, exercise, and rest. In the ready session, we measured the lactate level in blood, spectral edge frequency (SEF) of maximal voluntary isometric contraction (MVIC), and heart rate recovery (HRR). In the exercise session, the subject exercised gait on a slope of $15 \%$, a velocity of $4 \mathrm{~km} / \mathrm{h}$ and a time of $30 \mathrm{~min}$ with no restriction. In the rest session, the training group rested on the vibrating chair with vibration but control group rested on it with no vibration during $30 \mathrm{~min}$. After rest, we measured the lactate level and HRR to assess the recovery effect. We recommended all subjects to adopt the same posture during rest to minimize errors and all sessions were repeated 10 times over 5 weeks. The experimental environment was maintained with a constant room temperature at $20^{\circ} \mathrm{C}$ and humidity at $50 \%$ during all session in Fig. 1(b).

\subsection{Experimental protocol}

WBV and a control group without vibration. The experimental procedure progressed in sequence WBV loading was applied at a frequency of $10 \mathrm{~Hz}$ and amplitude of $5 \mathrm{~mm}$. During all protocols, the subjects lay down on the chair with their lumbar region extended at $120^{\circ}$ and knees flexed at $90^{\circ}$ in the platform, as shown in Fig. 1(c). The subjects were asked to keep the same posture.

Measurements were performed of the three parameters: lactate level in blood, SEF of MVIC, and HRR test. First, to observe recovery from fatigue, we measured the lactate level in blood using an Accutrend plus (Cobas, Ltd., USA). We disinfected the surface of skin first using alcohol and then drew blood. The test for lactate levels was performed before exercise, immediately after exercise, and after rest for $30 \mathrm{~min}$. Second, we measured SEF of MVIC in iliocostalis lumborum, rectus abdominis, rectus femoris, biceps femoris, tibialis anterior, and gastrocnemius using a Delsys Bagnoli-8 EMG system.

Specifically, we measured and analyzed SEF 50. The spectral edge frequency is a measure used in signal processing. The SEF 50 is the frequency below which $50 \%$ of the total power of a given signal in located. Measuring postures were flying, crunch, toe-up, heel-up, and squat. In the flying posture, the subject raised her waist maximally for the iliocostalis lumborum, but kept her lower limbs on the floor. The crunch posture is a common abdominal exercise and primarily works the rectus abdominis muscle. The subject was face up on the floor with knees bent and curling the shoulders towards the pelvis, and the hands can be beside the neck. In the toe-up, the subject placed her toes with bearing weight on the heel. In the heel-up, the subject placed her heels with bearing weight on the toes. The squat was initiated 

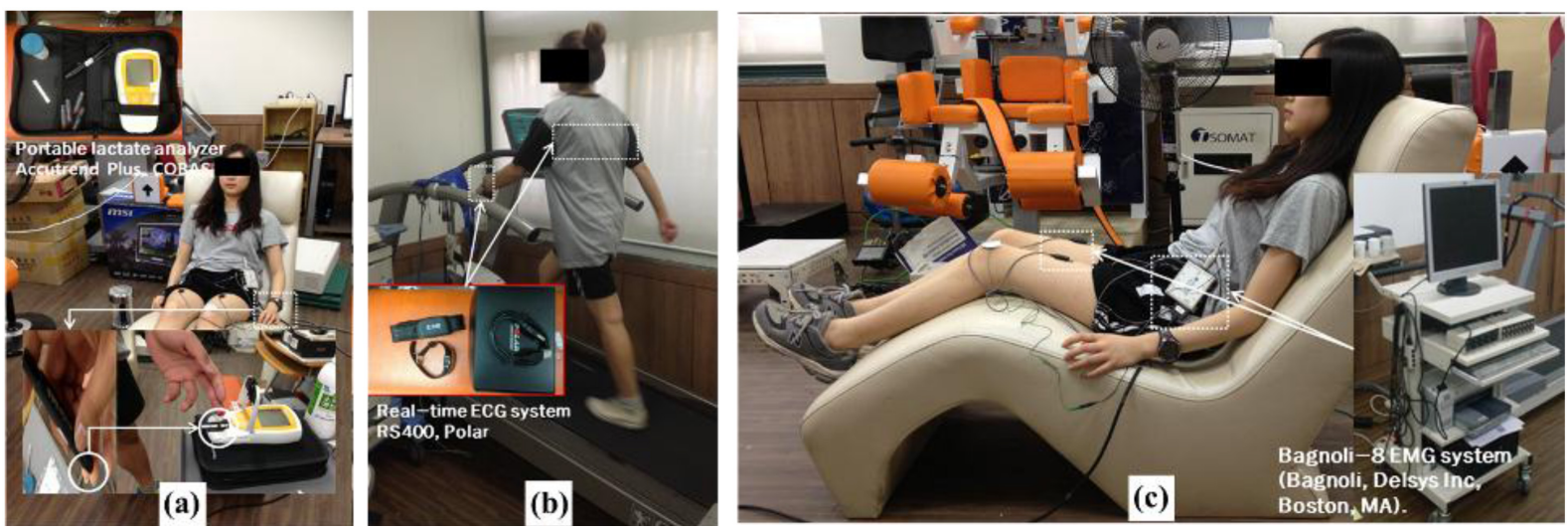

Fig. 2. Measurement performed in all sessions (a): measurement of blood lactate levels using a portable lactate analyzer, (b): measurement of HRR using a real-time ECG system, (c): measurement of SEF 50 for muscular activity using a real-time EMG system (Bagnoli, Delsys Inc., Boston, MA).

by moving the hips back and bending the knees and hips to lower the torso and accompanying weight, then returning to the upright position.

To observe the stabilizing body function, we tested the HRR parameter using a portable heart rate system (Polar RS400, POLAR, Ltd., USA) every $10 \mathrm{~min}$. The heart rate system was located at the center of chest and subjects wore a watch for checking the heart rate. We checked and recorded the variation in heart rate during all sessions every $10 \mathrm{~min}$. All sessions - test, exercise, and rest - were repeated 10 times, 2 days per week for 5 weeks (Fig. 2).

\subsection{Statistical analysis}

To observe recovery after fatigue, we measured lactate levels and HRR according to WBV use during rest. Statistical analyses were conducted using the SPSS software (ver. 18.0 for Windows; SPSS Inc., Chicago, IL, USA). Means and standard deviations (SD) were calculated for all variables. For each, the systematic change among the three trials was tested using a paired Student's t-test, and quantified from within-group differences. Post hoc Tukey tests, modified for repeated measures, were used to determine where significant differences occurred. Statistical significance was set at ${ }^{*} p \leqslant 0.05$.

\section{Results}

\subsection{Changes in blood lactate levels according to WBV in the rest session between the training and control groups}

The blood lactate level results showed that it decreased faster when providing WBV during rest in the training group than in the control group. Before exercise (ready session), the participants' lactate levels showed little few difference between groups at $0.11 \pm 0.02 \mathrm{mmol} / \mathrm{L}$. Immediately after exercise, the lactate level increased to $5.70 \mathrm{mmol} / \mathrm{L}$ (TG) and $5.76 \mathrm{mmol} / \mathrm{L}(\mathrm{CG})$ in both groups but with little difference between the groups. After the rest session, the lactate level in TG decreased from $7.21 \pm$ $0.55 \mathrm{mmol} / \mathrm{L}$ (end of exercise) to $1.63 \pm 0.21 \mathrm{mmol} / \mathrm{L}$ (end of rest), $77.45 \%$, for the group provided with whole body vibration. However, the lactate level in the control group showed a smaller decrease, from $7.15 \pm 0.73 \mathrm{mmol} / \mathrm{L}$ to $3.11 \pm 0.51 \mathrm{mmol} / \mathrm{L}, 56.61 \%$. Thus, there was a large difference in the reduction ratio, over $20 \%$, between the groups according to WBV (Fig. 3a). 


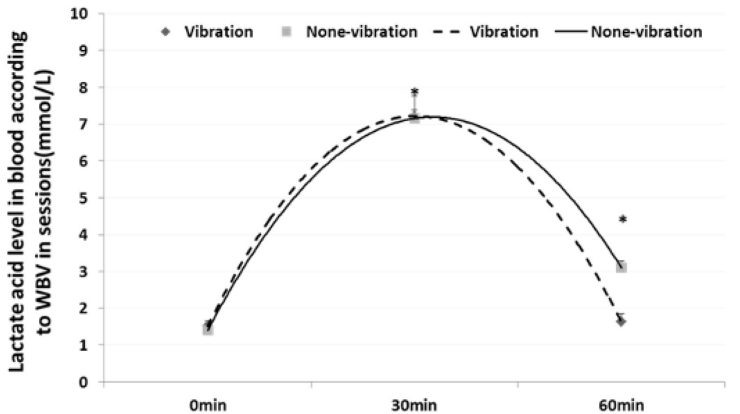

(a)

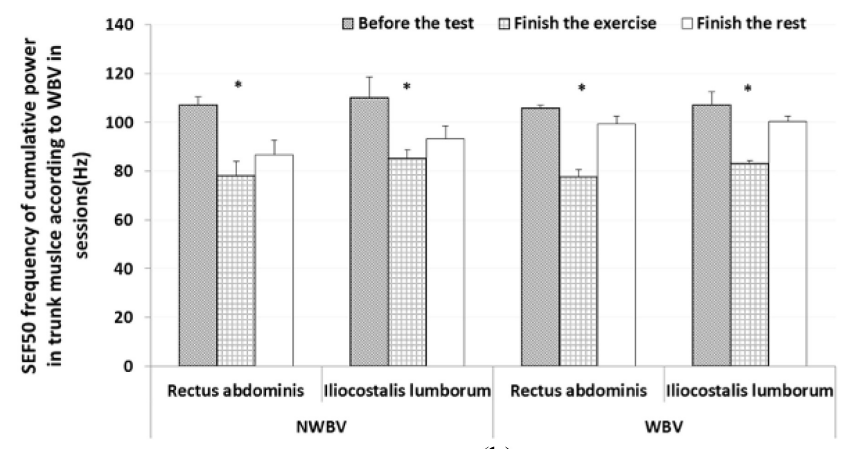

(b)

Fig. 3. (a) Variation in blood lactate levels according to WBV between groups (mean $\pm \mathrm{SD},{ }^{*} p<0.05$ ), (b) Results of SEF 50 frequency of cumulative power for observing muscle fatigue in trunk muscles according to WBV (mean $\pm \mathrm{SD},{ }^{*} p<0.05$ ).

\subsection{Changes in the SEF 50 of MVIC in muscles according to WBV in the rest session between the training and control groups}

We measured and analyzed SEF 50 of MVIC in muscles. Specifically, we assessed trunk muscles with the iliocostalis lumborum and rectus abdominis, femoral muscles with the rectus femoris and biceps femoris, and lower leg muscles with the tibialis anterior and gastrocnemius. The result of the rectus abdominis showed that the medial frequency was reduced at $26.75 \%$ (ready session: $105.83 \pm 1.21 \mathrm{~Hz}$, after exercise: $77.52 \pm 3.13 \mathrm{~Hz}$ ) in TG. With WBV, the medial frequency recovered to $28.18 \%$ (rest session: $99.37 \pm 3.15 \mathrm{~Hz}$ ) and muscular activity was at $93.89 \%$ in contrast with the ready session, for a recovery ratio at $159.14 \%$. However, the medial frequency also reduced at $27.09 \%$ (ready session: $107.20 \pm 3.21 \mathrm{~Hz}$, after exercise: $78.15 \pm 5.84 \mathrm{~Hz}$ ) in CG. The medial frequency in static rest recovered at $10.87 \%$ (rest session: $86.65 \pm 6.15 \mathrm{~Hz}$ ). This shows less recovery of muscular activity at $80.83 \%$ versus the ready session. The results of the iliocostalis lumborum muscle showed that the medial frequency reduced at $26.75 \%$ (ready session: $107.25 \pm 5.25 \mathrm{~Hz}$, after exercise: $83.15 \pm 1.16 \mathrm{~Hz}$ ) in TG and $22.71 \%$ (ready session: $110.25 \pm 8.21 \mathrm{~Hz}$, after exercise: $85.21 \pm 3.54 \mathrm{~Hz}$ ) in CG. After rest, the medial frequency recovered at $9.43 \%$ (rest session: $93.25 \pm 5.21 \mathrm{~Hz}$ ), including muscular activity at $84.58 \%$ (Fig. 3b).

The results for the rectus femoris showed that the medial frequency was reduced at $15.35 \%$ (ready session: $92.5 \pm 3.54 \mathrm{~Hz}$, after exercise: $78.3 \pm 2.54 \mathrm{~Hz}$ ) in TG. After the rest session, the medial frequency recovered at $15.58 \%$ by WBV (rest session: $90.5 \pm 1.21 \mathrm{~Hz}$ ) in TG. The muscular activity recovered at $97.83 \%$ versus the ready session with a higher recovery ratio at $290.01 \%$ than static rest in CG. The medial frequency also reduced at $14.69 \%$ (ready session: $93.9 \pm 1.22 \mathrm{~Hz}$, after exercise: $80.1 \pm 5.01 \mathrm{~Hz}$ ) in CG. The medial frequency during static rest recovered at 3.99\% (rest session: 83.3 $\pm 5.13 \mathrm{~Hz}$ ). That showed less recovery of muscular activity at $88.71 \%$ versus the ready session. The results of the biceps femoris muscle showed that the medial frequency decreased at $15.24 \%$ (ready session: $102.3 \pm 3.75 \mathrm{~Hz}$, after exercise: $86.7 \pm 3.36 \mathrm{~Hz}$ ) in TG and $22.71 \%$ (ready session: $100.8 \pm$ $3.56 \mathrm{~Hz}$, after exercise: $85.3 \pm 3.69 \mathrm{~Hz}$ ) in CG. After rest, the medial frequency recovered at $10.14 \%$ (rest session: $95.5 \pm 3.01 \mathrm{~Hz}$ ) including muscular activity at $93.35 \%$ but $88.71 \%$ with recovery at $3.99 \%$ (rest session $83.3 \pm 5.13 \mathrm{~Hz}$ ) (Fig. 4a).

The result of the tibialis anterior showed that the medial frequency reduced at $14.04 \%$ (ready session: $96.10 \pm 1.15 \mathrm{~Hz}$, after exercise: $82.60 \pm 3.21 \mathrm{~Hz}$ ) in TG. After the rest session with WBV, the medial frequency recovered faster at $10.04 \%$ (rest session: $90.90 \pm 2.54 \mathrm{~Hz}$ ) than static rest in CG and the 


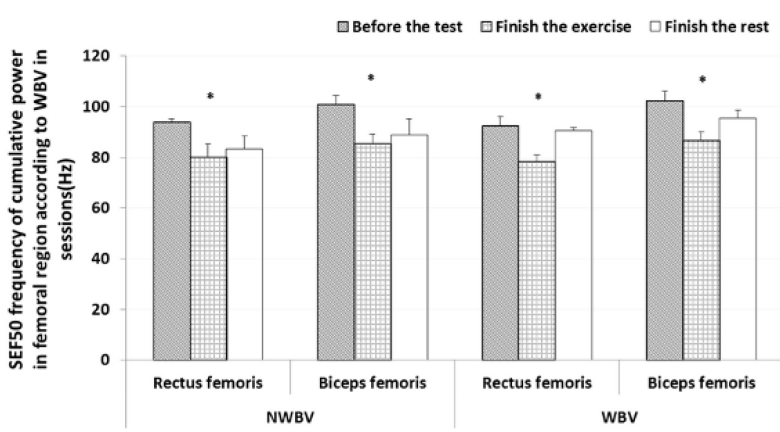

(a)

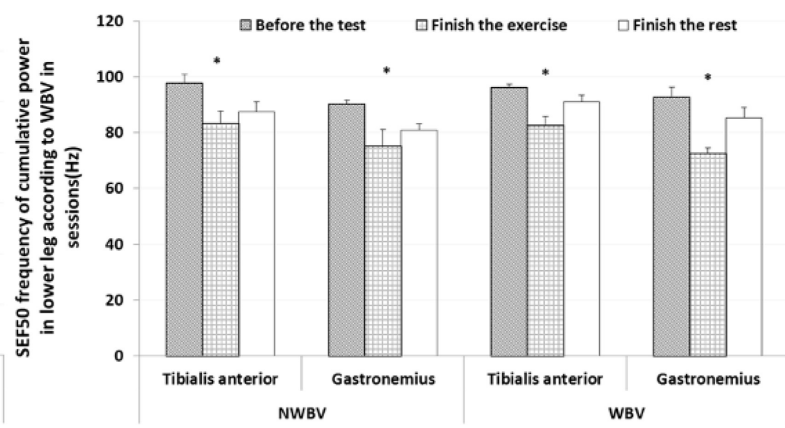

(b)

Fig. 4. Results of SEF 50 frequency of cumulative power for observing muscle fatigue according to WBV (mean $\pm \mathrm{SD},{ }^{*} p<$ 0.05) : (a) Femoral muscles, (b) Lower leg muscles.

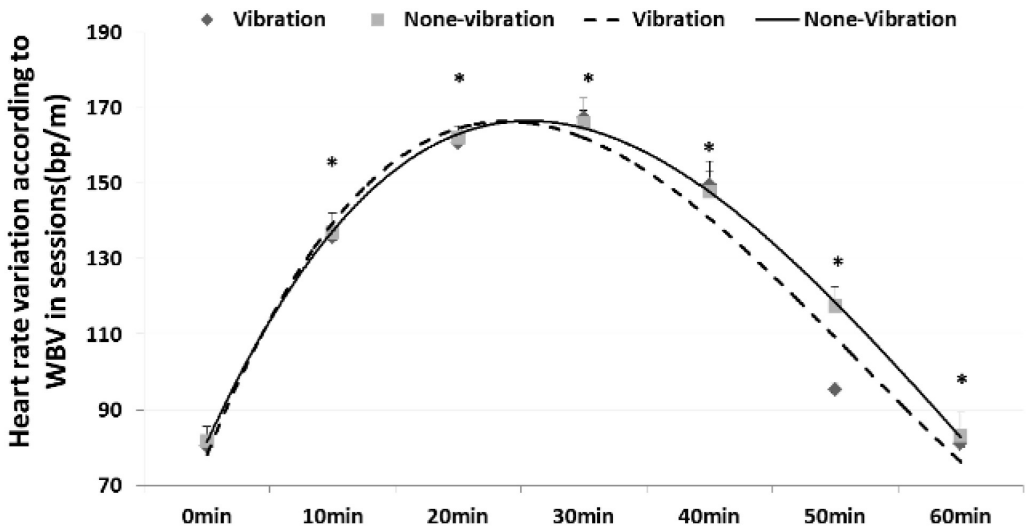

Fig. 5. Variation in heart rate according to WBV during rest after exercise between groups (mean $\pm \mathrm{SD},{ }^{*} p<0.05$ ).

muscular activity was $94.58 \%$ compared with the ready session including a higher recovery ratio at 92.07\%. However, the medial frequency in CG was reduced at $14.84 \%$ (ready session: $97.65 \pm 3.15 \mathrm{~Hz}$, after exercise: $83.15 \pm 4.54 \mathrm{~Hz}$ ) in CG. The medial frequency after static rest recovered at $5.23 \%$ (rest session: $87.50 \pm 3.56 \mathrm{~Hz}$ ). This showed a lower recovery ratio of muscular activity at $89.60 \%$ in CG. The results for the gastrocnemius showed that the medial frequency decreased at $21.79 \%$ (ready session: 92.7 $\pm 3.55 \mathrm{~Hz}$, after exercise: $72.50 \pm 2.16 \mathrm{~Hz}$ ) in TG and $16.89 \%$ (ready session: $90.15 \pm 1.54 \mathrm{~Hz}$, after exercise: $75.01 \pm 6.21 \mathrm{~Hz}$ ) in CG. After rest, the medial frequency recovered at $17.51 \%$ (rest session $85.20 \pm 3.87 \mathrm{~Hz}$ ) including muscular activity at $91.90 \%$ in TG but $89.73 \%$ with recovery at $7.86 \%$ (rest session $80.90 \pm 2.21 \mathrm{~Hz}$ ) (Fig. 4b).

\subsection{Changes in HRR according to WBV in the rest session between the training and control groups}

The HRR results showed that it recovered faster by providing WBV in TG than static rest in CG. In the ready session, there was little difference in HRR at $2.25 \pm 0.75 \mathrm{bp} / \mathrm{min}$ with $2.63 \%$ between the groups. During the exercise session, the HR of both groups increased to $108.33 \%$ (TG: $167.5 \pm 5.13 \mathrm{bp} / \mathrm{min}$, CG: $166.2 \pm 3.13 \mathrm{bp} / \mathrm{min}$ ) with little difference at $2.55-3.73 \%$ between groups (Fig. 5). There was little difference between groups in the first $10 \mathrm{~min}$ of the rest session. However, the results of HR showed 
different trends at $20 \mathrm{~min}$. In TG, HR decreased quickly to $95.4 \pm 2.01 \mathrm{bp} / \mathrm{min}$ with $43.04 \%$ in contrast to $117.5 \pm 5.21 \mathrm{bp} / \mathrm{min}$ with $29.30 \%$ in CG.

\section{Discussion}

Previous studies on WBV focused mostly on improvement in body condition, such as muscle strength, postural balance, flexibility, and coordination ability. The whole-body vibration could improve peripheral blood flow [21], pressure wave reflection [22], and leg arterial stiffness [23]. The whole body vibration caused a reflex neuromuscular stimulation [24] and, increased venous return as result of an active muscle pump [25]. However, most of studies only reported exercise effects without examining recovery effects. In this research, we investigated the recovery effect in terms of lactate level, medial frequency of muscular activity, and HRR according to WBV during rest after a gait exercise. The main findings were that WBV in rest after exercise significantly decreased fatigue in the human body, as evidenced by blood lactate levels, muscular fatigues, and heart rate changes. Furthermore, the results demonstrated that rest, applying to WBV after exercise, could elicit cool down effects of different kinds in these parameters.

We demonstrated that WBV in rest after exercise could improve the recovery in terms of lactate level, muscular fatigue, and HRR function. In the case of HRR, the provision of WBV at $10 \mathrm{~Hz}$ in a supine position significantly decreased fatigue in the human body by $13.74 \%$, in contrast to a previous study using WBV at $25 \mathrm{~Hz}$ [26]. We provided a supine condition as the rest posture to investigate HRR variation in contrast to seated or standing conditions in previous studies [27,28]. However, a higher heart rate within 5 min of active recovery following supramaximal and submaximal cycling exercise was likely attributable to the stimulatory influence of central commands [29]. Our research provided WBV during the rest session for active recovery using reflex neuromuscular reactions. We suggest that WBV frequency did not strongly affect the extent of HRR function but it maybe affect exposal posture. We thought that the faster reduction ratio of heart rate during rest session with WBV was due to increased venous return from active muscle pumps. During the rest session for recovery, parasympathetic reactivation occurred rapidly in the first minute of recovery and heart rate decreased gradually as parasympathetic tone was reactivated and sympathetic tone declinedc from exercise levels [30].

The results of lactate levels and SEF 50 supported that our hypothesis, like the HRR results. WBV during rest after exercise could provide a significantly faster recovery effect than routine rest. WBV may activate more efficient exercise recovery with continuous stimulus of blood vessels in the muscle. This stimulus could increase the use of lactate as energy for the heart and the activated muscles and cause the faster distribution of the lactate into liver. In this research, immediately after exercise (30 min), the results of lactate levels between groups showed little difference with an increase of 2.5 times each, but in the recovery session $(60 \mathrm{~min})$, the lactate level results in TG showed that WBV caused a faster recovery, by approximately three-fold, including a recovery ratio from fatigue at $92.71 \%$ in contrast with the reduction of $45.07 \%$ in CG. After exercise, WBV can provide positive effects; WBV stimulus reduced lactate levels quickly by increasing lactic acid oxidation levels by maintaining blood flow into muscles.

Our study supported two hypotheses that have been postulated to explain the possible physiological basis for the recovery effect of WBV in terms of changes in blood lactate levels, muscular fatigue, and HRR. The first has been linked to the WBV during the rest session after exercise, which may reduce muscle fatigue and stabilize the heart rate effectively. The second hypothesis is based on the irrelevancy of WBV to any positive effect in cool down function after exercise for providing faster reduction of lactate and improving HRR function. Future studies should examine more detailed vibration protocols 
in terms of frequency, amplitude, and exposure time to normalize the recovery effect of WBV with exercise intensity.

\section{Conclusion}

We estimated the recovery effect in terms of blood lactate levels, medial frequency of muscular activity, and HRR according to WBV during rest after gait exercise in young females. We suggest that WBV could be appropriate for exercise cool-down. The experimental results, which were consistent with the study hypothesis, showed that the WBV rest method could be used to successfully assess the efficient recovery effects on blood lactate, muscular fatigue, and HRR function. Moreover, the significantly improved recovery function observed in the young participants suggested that they could benefit more from this WBV rest method as part of cool down after exercise and that a recovery protocol could be developed for women, patients, and athletes.

\section{Acknowledgments}

This research project was supported by the Sports Promotion Fund of Seoul Olympic Sports Promotion Foundation from Ministry of Culture, Sports and Tourism in 2016 and partially supported Convergence Technology Development Project funded Korea Small and Medium Business Administration in 2014 (No.S2177266).

\section{Conflict of interest}

None to report.

\section{References}

[1] Kang SR, Jeong GY, Bae JJ, Min JY, Yu CH, Kim JJ, Kwon TK, et al. Effect of Muscle Function and Muscular Reaction of Knee Joint in the Twenties on the Whole Body Vibration Exercise. J. Korean Soc. Precis. Eng. 2013; 30(7); 762.

[2] Jung HJ, Lee JE, Oh JK, Effects of WBV Exercise on OPG, RANKL and BMD in Ovariectomized Rats. Korean. J. Sprots. Sci. 2007; 18(3); 10.

[3] Cardinale M, Wakeling J, Whole body vibration exercise: are vibrations good for you? Br. J. Sports. Med. 2005; 39(9); 585.

[4] Delecluse C, Roelants M, Verschueren S, Strength increase after whole body vibration compared with resistance training. Med. Sci. Sports. Exerc. 2003; 35(6); 1033.

[5] Seo SB, Kang SR, Yu CH, Min JY, Kwon TK, The Effect on Improvement of Muscle Strength Imbalance According to Load Deviation Protocol of Whole Body Vibration Exercise. J. Korean Soc. Precis. Eng. 2013; 30(10); 1095.

[6] Dolny DG, Reyes GF, Whole body vibration exercise: training and benefits. Curr. Sports. Med. Rep. 2008; 7(3); 152.

[7] Fagnani F, Giombini A, DiCesare A, Pigozzi F, DiSalvo V, The effects of a whole-body vibration program on muscle performance and flexibility in female athletes. Am. J. Phys. Med. Rehabil. 2006; 85(12); 956.

[8] Van Den Tillaar R, Will whole-body vibration training help increase the range of motion of the hamstrings? J. Strength. Cond. Res. 2006; 20(1); 192.

[9] Park DH, Hwang JH, Kim KH, Changes of metabolic and physiological variables to vibration frequency during whole body vibration exercise. Journal of Korea Physical Education Association for Girls and Women. 2008; 22(1); 39.

[10] Torvin SP, Kannus HS, Nen TA, Rvinen M, Pasanen S, Kontulainen TL, Jarvinen M, Jarvinen PO, Vuori I, et al. Effect of four-month vertical whole body vibration on performance and balance. Med. Sci. Sports. Exerc. 2002; 34(9); 1523. 
[11] Ko CY, Lee TW, Woo DG, Kim HS, Kim HS, Lee BY, Lim D, et al. Effect of whole body vibration on Osteroporotic Trabecular bone of rats - compared with the effect of Actonel. J. Korean Soc. Precis. Eng. 2008; 25(5); 148.

[12] Trans T, Aaboe J, Henriksen M, Christensen R, Bliddal H, Lund H, Effect of whole body vibration exercise on muscle strength and proprioception in females with knee osteoarthritis. Knee. 2009; 16(4); 256.

[13] Cardinale M, Wakeling J, Whole body vibration exercise: are vibrations good for you? Br. J. Sports. Med. 2005; 39(9); 585.

[14] Bazett-Jones DM, Finch HW, Dugan EL, Comparing the effects of various whole-body vibration accelerations on counter-movement jump performance. J. Sports. Sci. Med. 2008; 7(1); 144.

[15] Crewther B, Cronin J, Keogh J, Gravitational forces and whole body vibration: implications for prescription of vibratory stimulation. Phys. Ther. Sport. 2004; 5(1); 37.

[16] Cardinale M, Lim J, The acute effects of two different whole body vibration frequencies on vertical jump performance. Med. Sport. 2003; 56(4); 287.

[17] Adams JB, Edwards D, Serviette DH, Bedient AM, Huntsman E, Jacobs KA, Del Rossi G, Roos BA, Signorile JF, Optimal frequency, displacement, duration and recovery patterns to maximize power output following acute whole-body vibration. J. Strength. Cond. Res. 2009; 23(1); 237.

[18] Cochrane DJ, Stannard SR, Acute whole body vibration training increases vertical jump and flexibility performance in elite female field hockey players. Br. J. Sports. Med. 2005; 39(11); 860.

[19] Jacobs PL, Burns P, Acute enhancement of lower-extremity dynamic strength and flexibility with whole-body vibration. J. Strength. Cond. Res. 2009; 23(1); 51.

[20] Woods K, Bishop P, Jones E, Warm-up and stretching in the prevention of muscular injury. Sports. Med. 2007; 37(12); 1089.

[21] Lohman EB, Petrofsky JS, Maloney-Hinds C, Betts-Schwab H, Thorpe D, The effect of whole body vibration on lower extremity skin blood flow in normal subjects. Med. Sci. Monit. 2007; 13(2); CR71.

[22] Sanchez-Gonzalez MA, Wong A, Vicil F, Gil R, Park SY, Figueroa A, et al. Impact of passive vibration on pressure pulse wave characteristics. J. Hum. Hypertens. 2012; 26(10); 610.

[23] Wong AM, Sanchez-Gonzalez MA, Gil R, Vicil F, Park SY, Figueroa A, et al. Passive vibration on the legs reduces peripheral and systemic arterial stiffness. Hypertens. Res. 2012; 35(1); 126.

[24] Herrero AJ, Menendez H, Gil L, Martin J, Martin T, Garcia-Lopez D, Gil-Agudo A, Marín PJ, et al. Effects of wholebody vibration on blood flow and neuromuscular activity in spinal cord injury. Spinal Cord. 2011; 49(4); 554.

[25] Takahashi T, Hayano J, Okada A, Saitoh T, Kamiya A, Effects of the muscle pump and body posture on cardiovascular responses during recovery from cycle exercise. Eur. J. Appl. Physiol. 2005; 94(5-6); 576.

[26] Sanudoa B, César-Castillo M, Tejerob S, Nunesc N, de Hoyoa M, Figueroad A, Cardiac autonomic response during recovery from a maximal exercise using whole body vibration. Complement. Ther. Med. 2013; 21(4); 294.

[27] Crisafulli A, Scott AC, Wensel R, Davos CH, Francis DP, Pagliaro P, Coats AJ, Concu A, Piepoli MF, et al. Muscle metaboreflex-induced increases in stroke volume. Med. Sci. Sports. Exer. 2003; 35(2); 221.

[28] Perini R, Veicsteinas A, Heart rate variability and autonomic activity at rest and during exercise in various physiological conditions. Eur. J. Appl. Physiol. 2003; 90(3-4); 317.

[29] Takahashi T, Hayano J, Okada A, Saitoh T, Kamiya A, Effects of the muscle pump and body posture on cardiovascular responses during recovery from cycle exercise. Eur. J. Appl. Physiol. 2005; 94(5-6); 576.

[30] Imai K, Sato H, Hori M, Kusuoka H, Ozaki H, Yokoyama H, et al. Vagally mediated heart rate recovery after exercise is accelerated in athletes but blunted in patients with chronic heart failure. J. Am. Coll. Cardiol. 1994; 24(6); 1529. 